\title{
ELASTIC-PLASTIC MODE-II FRACTURE OF ADHESIVE JOINTS
}

\author{
Q. D. Yang ${ }^{1}$, M. D. Thouless ${ }^{1,2}$ and S. M. Ward ${ }^{3}$ \\ ${ }^{1}$ Department of Mechanical Engineering \& Applied Mechanics \\ ${ }^{2}$ Department of Materials Science \& Engineering \\ University of Michigan, Ann Arbor, MI 48109 \\ ${ }^{3}$ Scientific Research Laboratories \\ Ford Motor Company \\ Dearborn, MI 48121
}

\begin{abstract}
A numerical study of the elastic-plastic mode-II fracture of adhesive joints is presented in this paper. A traction-separation law was used to simulate the mode-II interfacial fracture of adhesively-bonded end-notched flexure (E.N.F.) specimens loaded in three-point bending, with extensive plastic deformation accompanying failure. The fracture parameters for the traction-separation law were determined by comparing the numerical and experimental results for one particular geometry. These parameters were then used without further modification to simulate the fracture of other E.N.F. specimens with different geometries. It was found that the numerical predictions for the loads and deformation were in excellent agreement with the corresponding experimental results.
\end{abstract}

Keywords: adhesive joints, fracture, plasticity, numerical modeling, mechanical testing

(Revised: May 2000) 


\section{Introduction}

Joining two materials by a third phase is of long-standing interest in a variety of industrial and technological applications, including traditional adhesive bonding, brazing and soldering, and composite materials. For elastic adhesive joints, in which the deformation of the adherends is dominated by elasticity during the fracture process, the conditions for fracture can be predicted using linear-elastic fracture mechanics (L.E.F.M). A substantial amount of work has been done in this area, and a detailed review of the appropriate mechanics has been given by Hutchinson and Suo (1992). However, the development of suitable analyses for the fracture of adhesive joints where the adherends deform plastically during the fracture process still remains incomplete because of the complications caused by the coupling between macroscopic plasticity in the adherends and the actual fracture process.

It has been demonstrated that the use of a traction-separation relation to characterize the fracture process is a very promising technique to deal with coupled plasticity and fracture (Hutchinson and Evans, 1999). A number of groups (Needleman, 1987; 1997, Tvergaard and Hutchinson, 1992; 1993; 1994, Wei and Hutchinson, 1997; 1998) have demonstrated that toughness is not the only controlling parameter when plasticity accompanies fracture: the cohesive stresses exerted at the crack-tip also play a very important role. Fracture can be simulated by using an embedded-process-zone (or cohesive-zone) model in which the toughness and peak cohesive stress are used as the two major parameters characterizing the fracture process. By incorporating such a model 
into nonlinear finite-element analyses which account for plasticity in the surrounding materials, the energy dissipated by plasticity can be separated from that absorbed during the decohesion process along the crack plane.

The present work is motivated by the authors' attempt to establish a general modeling approach to obtain quantitative predictions for the fracture of adhesive joints that are deforming plastically. The core of this approach is to use a cohesive-zone model to mimic the role that the adhesive layer plays during the deformation and fracture of the joints. The interfacial toughness and cohesive stresses are incorporated in the tractionseparation law of cohesive-zone elements that interact with the surrounding material. By doing this, the energy associated with fracture is completely separated out from the energy dissipated by gross plasticity in adherends. This approach has been successfully used to predict the mode-I fracture of various adhesive joints failing with extensive plastic deformation (Yang et al., 1999; 2000). An experimental procedure for determining the parameters of a suitable cohesive-zone model was proposed in these studies, and it was shown that numerical predictions based on these parameters were in excellent agreement with experimental results. While these studies demonstrated the success of the approach for mode-I fracture, establishing a general framework requires an understanding of mixedmode fracture. As a first step towards this goal, a study of pure mode-II fracture is described here. An extension to the general mixed-mode case, based on this work and the earlier mode-I studies, has recently been developed (Yang and Thouless, 2000). 
Three-point bending of adhesively-bonded, end-notched flexure (E.N.F.) specimens (Fig. 1) was chosen to provide the mode-II geometry. Stable crack growth occurs before the crack reaches the loading point in this type of specimen, and it provides an ideal case study for mode-II fracture. Furthermore, the test has previously been used to evaluate the mode-II fracture toughness of elastic adhesive joints based on L.E.F.M. concepts (Barret and Foschi, 1977; Chai, 1988). Chiang and Chai (1994) studied the plasticity in the adhesive layer near the static crack-tip of elastic E.N.F adhesive joints using large-strain finite-element analyses. The deformation of the adhesive layer in the crack-tip region is essentially dominated by shear (with some compressive normal stresses at the crack-tip). However, only elastically-deforming adherends were considered in these earlier studies. When plasticity occurs, additional complications arise not only from the coupling effects between the fracture process and plasticity in adherends, but also from the geometric non-linearities caused by large deformations and rotations of the specimens. Therefore, a full nonlinear numerical analysis incorporating an appropriate cohesive-zone model is necessary for modeling the fracture of such joints.

In this paper, a mode-II fracture problem is analyzed by adapting the technique previously used with success to model mode-I fracture (Yang et. al., 1999). As shown in Fig. 2, the shear traction-separation relationship of a mode-II cohesive zone is characterized by $\Gamma_{\text {IIo }}$, the work of separation per unit area of crack growth (equal to the area under the curve), the peak shear stress supported by the bonding tractions, $\hat{\tau}$, and two shape parameters $\delta_{t 1} / \delta_{t c}$ and $\delta_{t 2} / \delta_{t c}$. Numerical experiments confirmed that $\Gamma_{\text {IIo }}$ and $\hat{\tau}$ 
are the two key parameters that dominate the numerical simulations; the two shape parameters are less important. Therefore, in the work that follows, the two key parameters are first determined and then used to predict fracture. Comparisons between the numerical predictions and the experimental observations of crack propagation, deformation and fracture are used to validate the numerical technique.

\section{Experiments}

\subsection{Torsion test of adhesively-bonded butt joints}

Torsion tests of adhesively-bonded butt joints were conducted to obtain the shear properties of an adhesive layer (XD4600, from Ciba Specialty Chemicals) with a bondline thickness of $0.25 \mathrm{~mm}$. This is identical to the thickness used subsequently for the E.N.F. specimens. This is essential because it is expected that the shear properties of adhesive layers depend upon the bond-line thickness (Chai, 1993). The butt joint was made of two solid aluminum cylindrical rods with a diameter of $12.7 \mathrm{~mm}$ (see the inset of Fig. 3a). The two rods were bonded together by the adhesive and carefully aligned by clamping them to a V-box during curing. The bond-line thickness was controlled by using uniform-sized silica spheres with a diameter of $0.25 \mathrm{~mm}$ as spacers. Subsequent measurements confirmed that the actual thickness was indeed $0.25 \mathrm{~mm}$ after the specimens were cured.

The shear test was performed on a torsion testing machine with a loading rate of $0.05 \mathrm{rad} / \mathrm{min}$. The gauge length of the sample was $98 \mathrm{~mm}$. In addition, an aluminum rod cut from the same batch and with the same gauge length was tested independently so that 
the elastic strain in the aluminum rods could be eliminated from the butt-joint test data. Curves of applied torque against rotation were recorded. After subtraction of the elastic rotation in the aluminum rods, a curve of applied torque versus relative rotation angle across the adhesive layer was obtained. This is shown as a solid line in Fig. 3(a). A numerical analysis using ABAQUS (5.8) with axisymmetric elements was performed to back out the shear stress-strain curve of the adhesive. The resultant curve is shown in Fig.3(b). The results of the numerical simulation of the torsion test based upon this constitutive relation are shown as a dashed line in Fig. 3(a). The shear stress-strain curve for the adhesive given in Fig. 3(b) provided guidance in the subsequent determination of the mode-II fracture parameters used in the cohesive-zone model.

\subsection{Three-point bending test of adhesively-bonded E.N.F. specimens}

Edge-notched flexure specimens were fabricated using $20 \mathrm{~mm}$ wide coupons cut from aluminum sheets (5754 alloy from Alcan Rolled Products Company) of different thicknesses varying between $1.6 \mathrm{~mm}$ and $3.0 \mathrm{~mm}$. The true stress-strain curve of the aluminum, which is essential for the numerical simulation to be introduced in next section, was obtained from standard uniaxial tensile tests and is shown in Fig. 4. The coupons were bonded by a layer of the commercial adhesive XD4600. The adhesive layer was kept at a uniform thickness of $0.25 \mathrm{~mm}$ using silica spheres as spacers. An initial crack along one of the adhesive/aluminum interfaces was introduced by inserting a strip of Teflon ${ }^{\circledR}$ tape into the interface before applying the adhesive. After curing, any excess adhesive was removed by careful filing and polishing of the specimen edges. To facilitate 
experimental observations, straight lines were cut vertically across the bond-lines using a sharp razor.

The specimens were placed over a test fixture consisting of two supporting cylinders. A third cylinder connected to the load cell of a tensile machine was used to bend the sample at its mid-point (Fig. 1). The span between the two supporting cylinders was $60 \mathrm{~mm}$, and the initial crack length was $20 \mathrm{~mm}$. To reduce possible frictional effects along the cracked surfaces, a glass fiber with a diameter of $0.4 \mathrm{~mm}$ was inserted as a spacer between the adherends at the crack mouth. The force and relative displacement between the center loading point and the supports were recorded as the specimens were deformed. The deflection measurements were made using a highresolution CCD camera. This camera was also used to monitor the crack propagation and the deformation of the adhesive layer near the crack-tip. A series of tests were performed for each thickness of aluminum. Each test was performed at room temperature, with a cross-head velocity of $2 \mathrm{~mm} / \mathrm{min}^{1}$

For all the specimens tested in this paper, the fracture was purely interfacial and the crack propagated along the interface between the adhesive layer and the compressive adherend. $^{2}$ The deformation of a $2.3 \mathrm{~mm}$ thick specimen immediately after the crack began to grow is shown in Fig. 5(a), and a magnified view of the crack-tip region is shown in Fig. 5(b). Figure 5(a) indicates the extent of frictional sliding between the sample and

\footnotetext{
${ }^{1}$ Experimental results show that there is no discernable difference between the load-displacement curves of samples tested at displacement rates of $0.2 \mathrm{~mm} / \mathrm{min}$ and $200 \mathrm{~mm} / \mathrm{min}$.
} 
the supports. The lines at the ends of the sample indicate the initial location of the contact points between the supports and the sample. The scribe marks in Fig. 5(b) show that the nominal shear strain across the bond-line is about $40 \%$ at a distance approximately twice the adhesive thickness ahead of the crack-tip. An even larger local strain is expected in the immediate vicinity of the crack-tip, as also observed by Chai (1992). The full extent of the plastic zone, measured from the crack-tip to the farthest point ahead of the tip which shows visible straining, was about 20 times the bond-line thickness.

\section{Numerical simulations and comparisons to experimental results}

Numerical calculations were performed to simulate the three-point bending tests. These calculations incorporated a cohesive-zone model within elastic-plastic finiteelement calculations using the ABAQUS code (version 5.8). Except for the contact regions between the glass fiber and the adherends, where three-point elements were used in order to obtain a finer mesh, four-point bi-linear elements were used for the adherends. Reduced integration was used for all these elements in order to prevent possible shearlocking effects. Large-strain and large-rotation conditions were considered, with the von Mises yield criterion and an isotropic strain-hardening model being used to simulate the mechanical behavior of the adherends. Two-dimensional analyses were used for the sake of numerical efficiency. It should be noted that plane-stress elements provided a better approximation to this geometry than plane-strain elements. Since this is not an obvious

\footnotetext{
${ }^{2}$ The crack always kinked into the interface immediately after it started to grow even if it was initiated at the mid-thickness of the adhesive layer.
} 
point, a more detailed discussion is given in the Appendix. Another important feature of this study was the use of contact elements to simulate the sliding between the supporting cylinders and the adherends, and between the glass fiber and the adherends. As can be seen from Fig. 5(a), there was fairly extensive sliding between the supporting cylinders and the adherends; this resulted in a decrease in the effective span length as the specimens deformed. Numerical experiments showed that failure to use contact elements between the supporting cylinders and adherends could result in as much as a $15 \%$ reduction in the predicted load. Therefore, it was deemed essential to use contact elements to allow the modeling of the sliding between the supporting cylinders and adherends, and between the glass fiber (spacer) and adherends.

In the numerical calculations, the adhesive layer was replaced by user-defined elements that simulated the appropriate traction-separation relationship of a mode-II cohesive-zone model. As described earlier, the work of separation per unit area, $\Gamma_{\text {IIo }}$, and the peak shear stress supported by the interface, $\hat{\tau}$, are the most important parameters that need to be determined for such a model. Systematic numerical experiments confirmed that the two shape parameters are less important, and they were chosen to be constants of $\delta_{t 1} / \delta_{t c}=0.15$ and $\delta_{t 2} / \delta_{t c}=0.5$ for all the simulations. The critical shear displacement, $\delta_{t c}$, is fixed by the choice of the shape parameters, $\Gamma_{\text {IIo }}$ and $\hat{\tau}$, since $\Gamma_{\text {IIo }}=0.5 \hat{\tau}\left(\delta_{t c}-\delta_{t 1}+\delta_{t 2}\right)$.

The three-point bending tests showed that the local strain in crack-tip region was at least $40 \%$. Therefore, it is evident from Fig. 3(b) that the peak shear stress can be 
taken as $\hat{\tau}=35 \mathrm{MPa}$. The mode-II joint toughness, $\Gamma_{\text {IIo }}$ was then determined to be $5.4 \mathrm{~kJ} / \mathrm{m}^{2}$ by using different values of $\Gamma_{\text {IIo }}$ to predict load-deflection curves for the $2.3 \mathrm{~mm}$ specimen, and finding the best fit to the associated experimental results. The resultant curve using these two parameters is shown in Fig. 6 together with a typical experimental curve. The oscillation in the numerical curve is caused by the difficult convergence procedure for the contact elements between the glass fiber and the adherends. The maximum loads in both the experimental and numerical curves correspond to the points at which the crack began to grow. The crack was observed to propagate steadily as the displacement was increased, but under a gradually decreasing load. It can be seen that the numerical calculation does an excellent job of reproducing the entire deformation history both before and after the beginning of crack growth. As a contrast to the numerical calculations using cohesive-zone elements, the results of a finite-element calculation using the continuum properties of the adhesive with no failure criterion are superimposed on Fig. 6. While such a calculation captures the deformation behavior until the crack begins to grow, the absence of a failure criterion renders it invalid after this point. However, the comparison shows that, in this particular case, the cohesive tractions are basically mimicking the deformations of the adhesive layer.

After being validated using the $2.3 \mathrm{~mm}$-thick samples, these values of $\hat{\tau}=35 \mathrm{MPa}$ and $\Gamma_{\text {IIo }}=5.4 \mathrm{kJm}^{-2}$ were then used without any further modification to simulate the fracture of other specimens with different thicknesses of the aluminum. The numerical predictions for the applied loads and deflections are shown as solid lines in 
Fig. 7. The shaded areas in this figure indicate the ranges of the corresponding experimental data. The numerical results are in excellent agreement with the experimental results; they not only reproduce the magnitudes of the forces, but also capture some of the nuances of the shapes of the load-deflection curves. However, since plane-stress conditions were assumed in all the simulations, it is not surprising to see that the numerical results are closer to the lower bounds of the experimental data.

The numerical prediction for the global deformation of a $2.3 \mathrm{~mm}$ thick specimen at the point when the crack begins to grow is shown in Fig. 8(a). A magnified view of the deformation and shear stress distribution near the crack-tip is shown in Fig. 8(b). A comparison between these numerical predictions and the experimentally-observed deformation (Fig. 5a) shows excellent agreement between the two. The predicted size of the plastic zone can be inferred from the extent of the shear deformation shown in Fig. 8(b); at approximately 20 times the bond-line thickness. This again agrees with the experimental observations (Fig. 5b).

\section{Discussion}

The pure mode-II interfacial toughness of the joint $\left(\Gamma_{\text {IIo }}=5.4 \mathrm{kJm}^{-2}\right)$ is much higher than the associated interfacial mode-I value of $\Gamma_{\mathrm{Io}}=1.0 \mathrm{kJm}^{-2}$ (with a peak normal cohesive stress $\hat{\sigma}=60 \mathrm{MPa}$ ), which was determined from wedge-induced fracture tests of double-cantilever beams bonded by an identical thickness of the adhesive (Yang and 
Thouless, 2000). ${ }^{3}$ This demonstrates that the fracture is highly mode-dependent. Similar results were also observed by Chai in his systematic studies of the fracture of elastic adhesive joints (Chai, 1988; 1992). These studies showed that, except for extremely thin adhesive layers $(<10 \mu \mathrm{m})$, the typical value of $\Gamma_{\text {IIo }}$ was about an order of magnitude higher than the corresponding $\Gamma_{\text {Io }}$ value. A major contribution to the high value of $\Gamma_{\text {IIo }}$ is the higher capability of the adhesive layer to deform plastically in response to shear. In the interfacial mode-I fracture tests, the maximum tensile strain across the adhesive layer before fracture was observed to be only about $8 \%$. The maximum shear strain observed in the torsion tests of the butt joints was about $60 \%$ (Fig. 3b). Therefore, much more energy is dissipated in the adhesive layer under mode-II loading than under mode-I loading. A rough estimate for the values of the mode-I and mode-II toughnesses can be obtained from the product of the critical displacements and cohesive stresses. For a $0.25 \mathrm{~mm}$ thick adhesive layer, the data given above would indicate a mode-I toughness of about $1 \mathrm{kJm}^{-2}$ and a mode-II toughness of about $5 \mathrm{kJm}^{-2}$. The large difference in the cohesive-zone parameters for mode-I fracture and mode-II fracture emphasizes the need for mode-dependent traction-separation relations to analyze mixed-mode fracture. This work has now been completed and will appear elsewhere (Yang and Thouless, 2000).

\section{Conclusions}

A pure mode-II cohesive-zone model has been used to simulate the elastic-plastic fracture of adhesively-bonded, end-notched flexure specimens loaded by three-point

\footnotetext{
${ }^{3}$ The value for $\Gamma_{\text {Io }}$ quoted here is for interfacial failure. A value of $\Gamma_{\text {Io }}=1.4 \mathrm{~kJ} / \mathrm{m}^{2}$ and $\hat{\sigma}=100 \mathrm{MPa}$ was
} 
bending. The fracture parameters of the model were determined by matching the numerical results with the associated experimental results. These parameters were then used without any further modifications to predict the fracture of other adhesive joints with different thicknesses of adherends. The numerical predictions were in excellent agreement with the associated experimental results. Therefore, it has been demonstrated that it is possible to predict the loads associated with crack growth, and to model the entire deformation history of plastically-deforming adhesive joints loaded in shear.

\section{Acknowledgments}

This work was supported by NSF Grant CMS-9624452 and Ford Motor Company. The authors would also like to express their appreciation to Prof. A. Waas and Mr. C. S. Yerramalli in the Dept. of Aerospace Engineering of University of Michigan for their help in the torsion test.

found for mode-I fracture within the adhesive layer (Yang et al., 1999). 


\section{References}

ABAQUS Finite Element Analysis Code and Technical Manual, Version 5.8, Hibbit, Karlsson and Sorensen Inc., 1998

Barret, J. D. and Foschi, R. O., Engineering Fracture Mechanics, 9 (1977) 371-378

Chai, H., International Journal of Fracture, 37 (1988), 137-157.

Chai, H., International Journal of Fracture, 58 (1992), 223-239.

Chai, H., Journal of Materials Science, 28 (1993), 4944-4956

Chiang, M. Y. M. and Chai, H., International Journal of Solids and Structures, 31 (1994), 2477-2490.

Hutchinson, J. W. and Evans, A. G., Harvard University, Division of Engineering \& Applied Sciences, Report MECH-351, 1999.

Hutchinson, J. W. and Suo Z., Advances in Applied Mechanics, 29, (1992), 63-191

Needleman, A., Journal of Applied Mechanics, 54 (1987), 525-531.

Needleman, A. Computational Mechanics, 19 (1997), 463-469.

Tvergaard, V. and Hutchinson, J. W., Journal of the Mechanics and Physics of Solids, 40 (1992), 1377-1397.

Tvergaard, V. and Hutchinson, J. W., Journal of the Mechanics and Physics of Solids, 41 (1993), 1119-1135.

Tvergaard, V. and Hutchinson, J. W., Philosophical Magazine A, 70 (1994), 641-656.

Wei Y. and Hutchinson, J. W., Journal of the Mechanics and Physics of Solids, 45 (1997), 1137-1159.

Wei Y. and Hutchinson, J. W., International Journal of Fracture, 93 (1998), 315-333. 
Yang, Q. D., Thouless, M. D. and Ward, S. M., Journal of the Mechanics and Physics of Solids, 47 (1999), 1337-1353.

Yang, Q. D., Thouless, M. D. and Ward, S. M., Journal of Adhesion, 72 (2000), 115-132.

Yang, Q. D., and Thouless, M. D., submitted to International Journal of Fracture, (2000). 


\section{Appendix}

While a full 3-D calculation is required for a rigorous analysis of this problem, a 2-D approximation was used in the interests of numerical efficiency. Plane-stress elements were used in these calculations despite the fact that, at first glance, the samples might appear to be under plane-strain conditions. The reason that plane-stress elements proved to be more useful in this particular study is that the adhesive layer is much more compliant than the aluminum adherends, and does not provide the constraint necessary for plane-strain deformation of the specimen. To demonstrate this point, a three-point bend test was performed on an adhesive joint made from $2.0 \mathrm{~mm}$ thick aluminum coupons with no pre-crack. Figure A1 shows the deformation in both the adherends and the adhesive. The relative displacements between the upper and lower beams, which result from shear deformation in the adhesive layer, are clearly seen in this figure. These displacements allow the two beams to deform in a relatively "independent" fashion-different from the predictions of simple beam theory. The stiffness of the composite beam is much lower than that of a solid beam with the same thickness.

This point is further demonstrated by the force versus deflection curves shown in Fig. A2. In this figure, the experimental results are shown by the solid line. Numerical results of 2-D plane-stress, 2-D plane-strain and 3-D finite-element analyses using a continuum model for the adhesive (not a cohesive-zone model) are also shown in this figure. It is evident that the 2-D plane-stress and 3-D $\mathrm{D}^{4}$ results were in agreement with the

\footnotetext{
${ }^{4}$ In this analysis, a relatively coarse mesh was used due to the limited memory size of the computers. Therefore, the 3-D results should be regarded as the upper limit of the actual load-displacement curve.
} 
experimental data, while the 2-D plane-strain assumption considerably over-estimated the beam stiffness. 


\section{Figure Captions}

Figure 1 Test configuration of an end-notched flexure (E.N.F.) specimen. The span, $2 L$, is $60 \mathrm{~mm}$, and the initial crack length, $a$, is $20 \mathrm{~mm}$.

Figure 2 The traction-separation law used in the numerical analyses. It is characterized by a work of separation per unit area, $\Gamma_{\text {IIo }}$, a peak shear stress, $\hat{\tau}$, and two shape parameters $\delta_{t 1} / \delta_{t c}$ and $\delta_{t 2} / \delta_{t c}$.

Figure 3 (a) Experimental curve of applied torque versus relative rotation across the adhesive layer of butt joints (solid line). Also shown in this figure is the numerical results predicted by the constitutive properties shown in Fig. 3(b) (dashed line).

(b) Constitutive properties of the adhesive XD4600 backed out from the torsion test of adhesively-bonded butt joints.

Figure 4 The true stress-strain relation of the adherend material (5754 aluminum alloy).

Figure 5 (a) Micrograph of a deformed $2.3 \mathrm{~mm}$ thick edge-notched flexure sample at the point at which the crack has just begun to grow.

(b) A magnified view of the deformations in the vicinity of the crack-tip.

Figure 6 Load (per unit width) for a $2.3 \mathrm{~mm}$ thick edge-notched flexure specimen plotted as a function of the deflection at the mid-point of span. Three 
curves are compared: (i) experimental data, (ii) numerical results of a cohesive-zone model with $\hat{\tau}=35 \mathrm{MPa}$ and $\Gamma_{\text {IIo }}=5.4 \mathrm{kJm}^{-2}$, and (iii) numerical results using a continuum model for the adhesive layer with no failure criterion.

Figure 7 Load (per unit width) plotted as a function of the deflection at the midpoint of the span for 1.6, 2.0, 2.3 and $3.0 \mathrm{~mm}$ thick, edge-notched flexure specimens. The shaded areas show the range of experimental results. The solid lines show the results of numerical calculations with $\hat{\tau}=35 \mathrm{MPa}$ and $\Gamma_{\text {IIo }}=5.4 \mathrm{kJm}^{-2}$.

Figure 8 (a) Numerical predictions for the deformation and the von Mises stress distribution in a $2.3 \mathrm{~mm}$ edge-notched flexure sample at the point at which the crack has just begun to grow.

(b) Numerical predictions for the distribution of shear stresses and for the shear displacements across the interface.

Figure A1 The relative deformation across the adhesive layer in an adhesively-bonded beam made with $2.0 \mathrm{~mm}$ thick aluminum adherends and tested in threepoint bending. The span was $60 \mathrm{~mm}$ between the outside supports. (The shear displacements disappeared at the central loading point to the right of the figure.) 
Figure A2 Numerically-predicted and experimentally-observed results for the load (per unit width) needed to deform an adhesively-bonded beam made with 2.0mm thick aluminum adherends and tested in three-point bending (with a span of $60 \mathrm{~mm}$ between the outside supports). The numerical results were obtained using 2-D plane-stress, 2-D plane-strain and 3-D finite-element elements with a continuum model for the adhesive. 


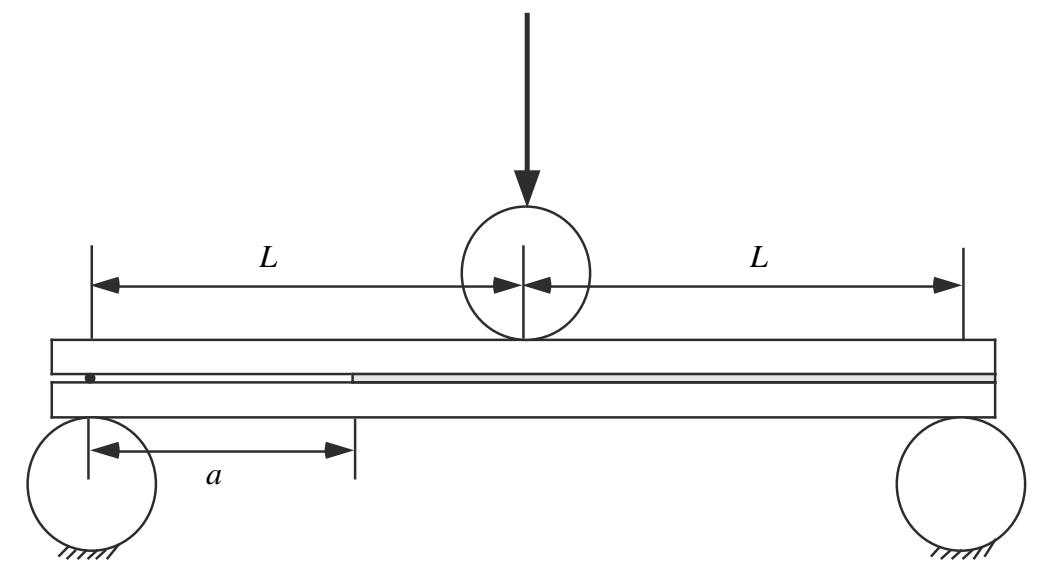

Fig. 1 


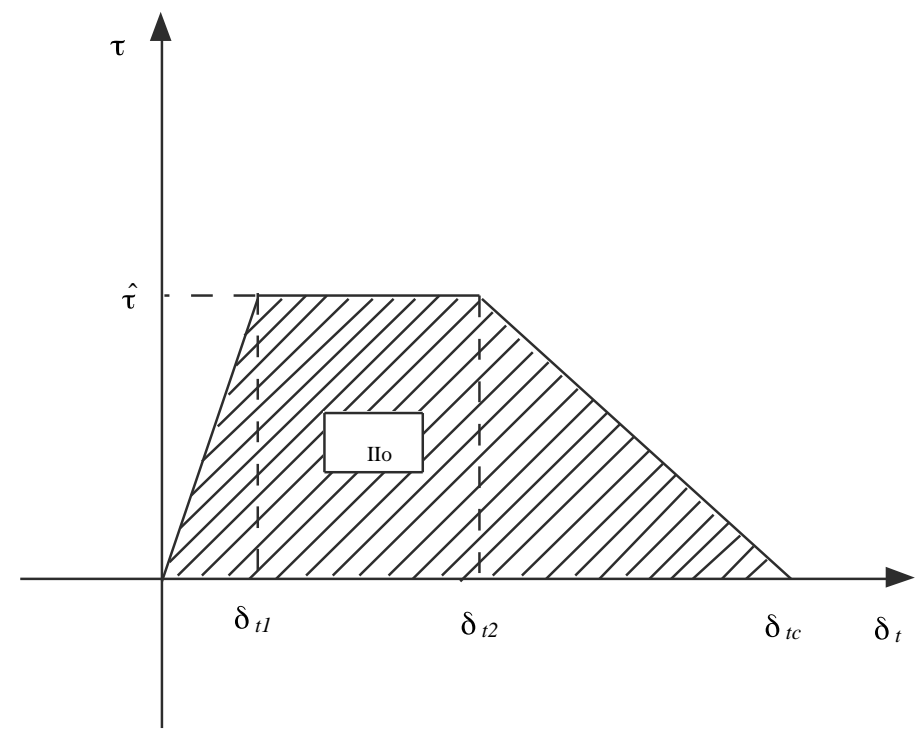

Fig. 2 


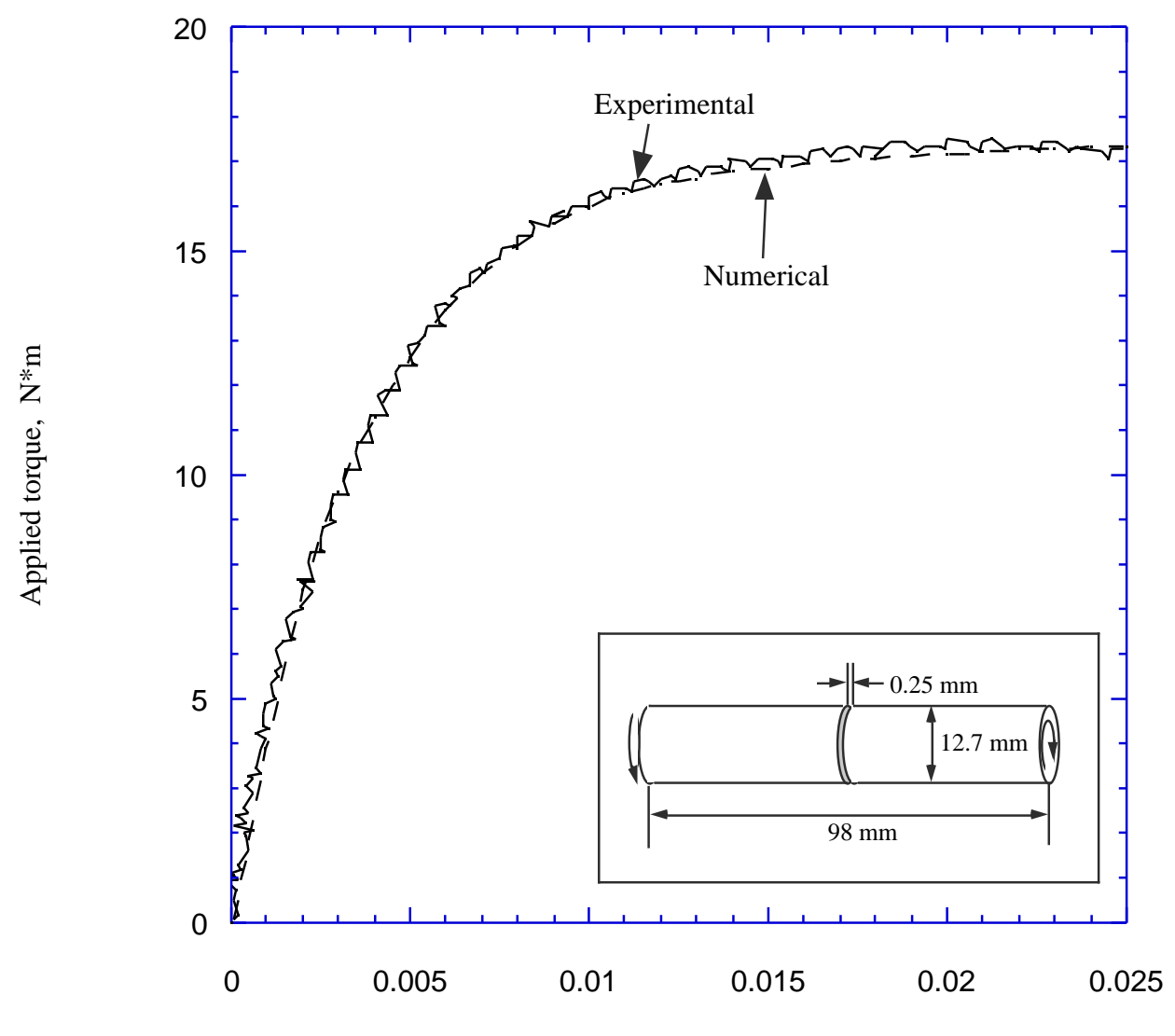

Relative rotation across the adhesive layer, radius

Fig. 3(a) 


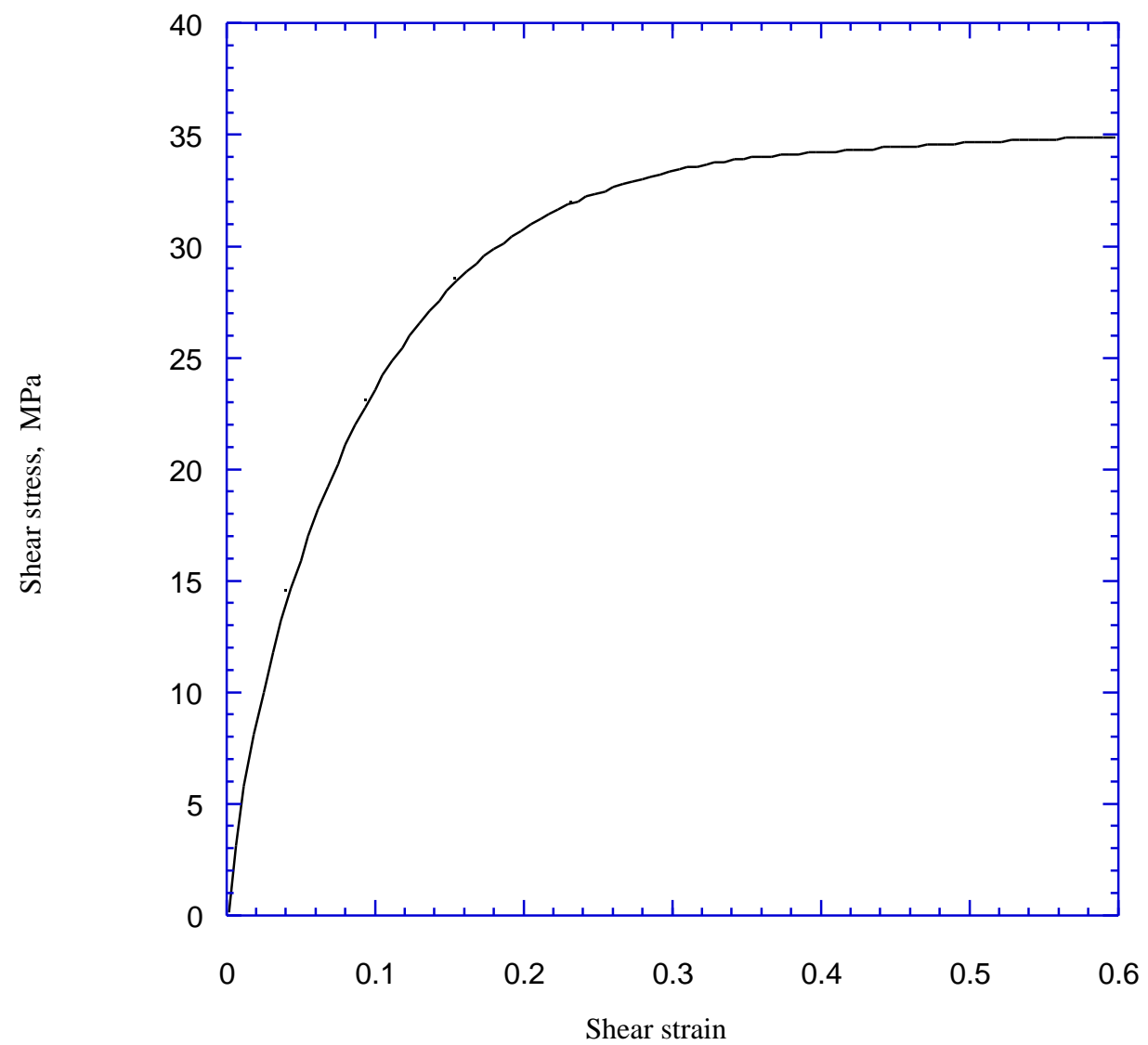

Fig. 3(b) 


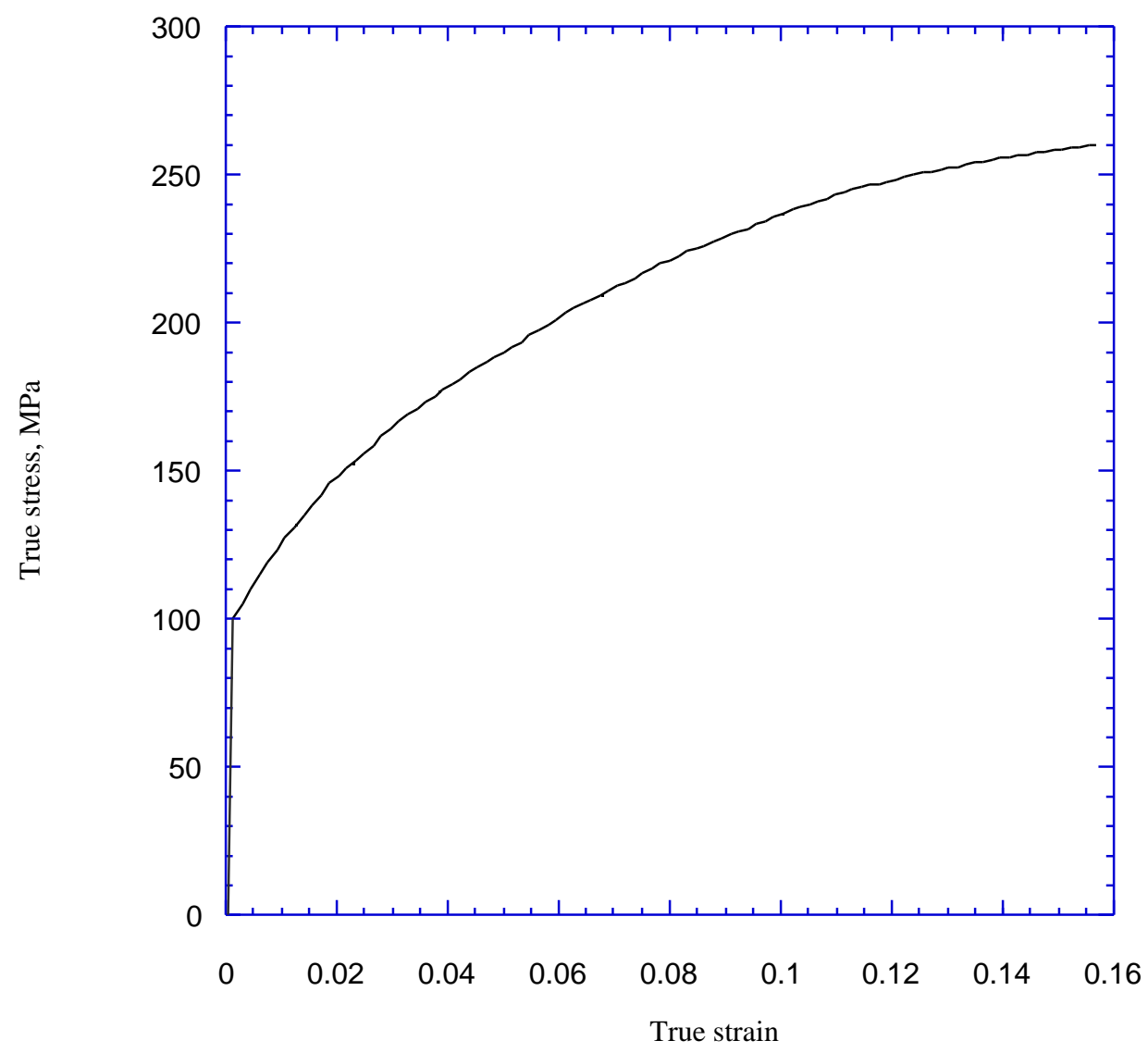

Fig. 4 


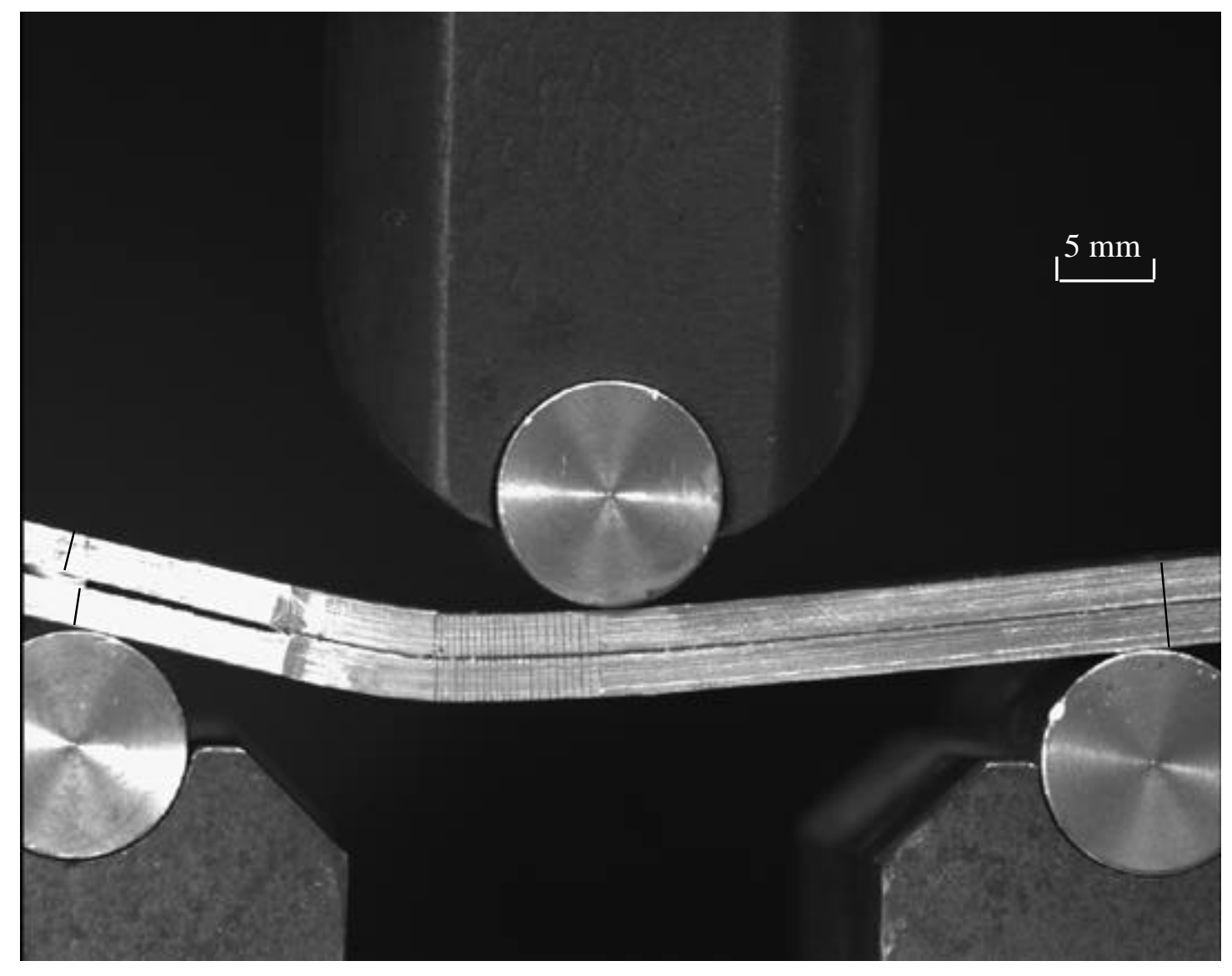

Fig 5 (a) 


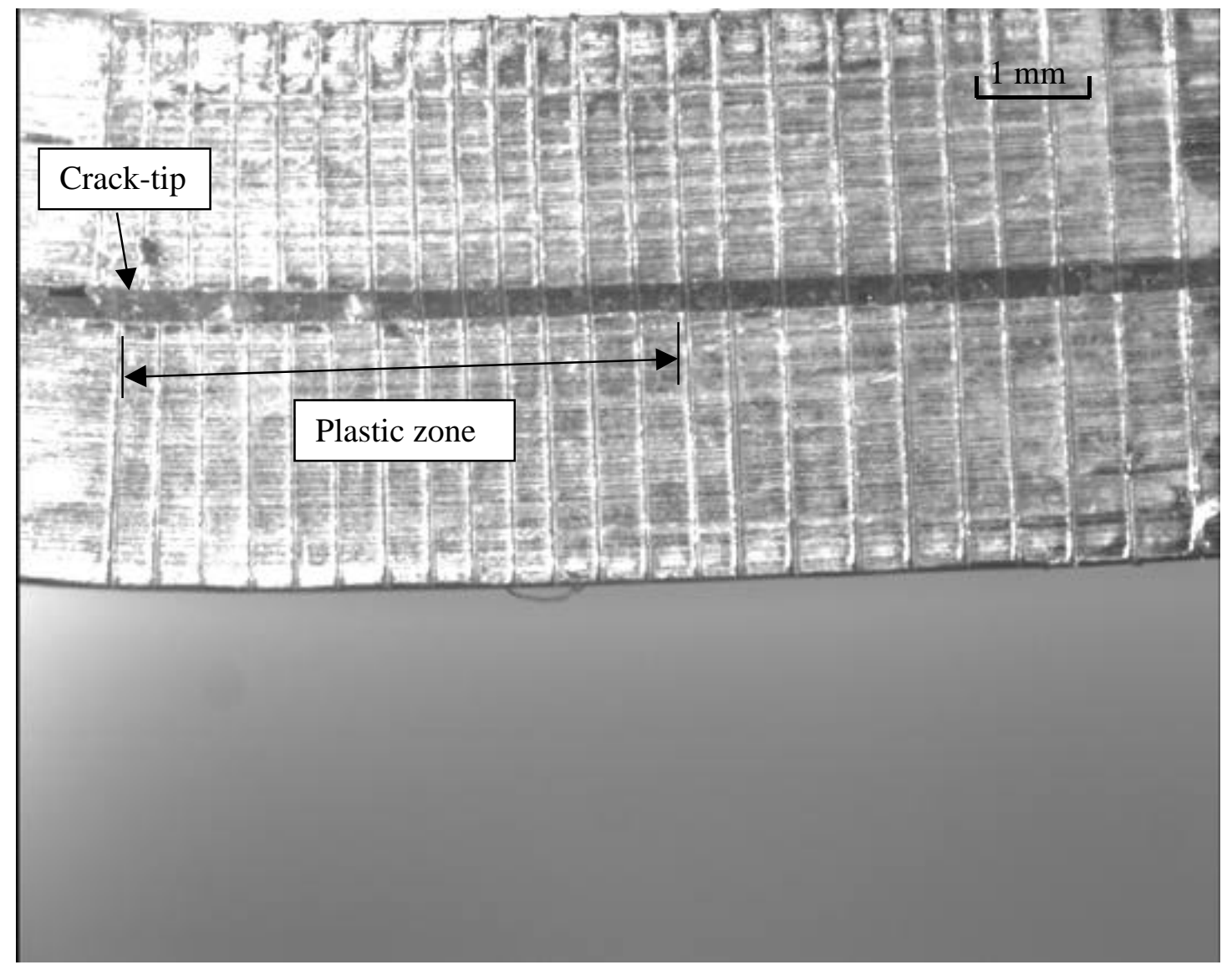

Fig. 5(b) 


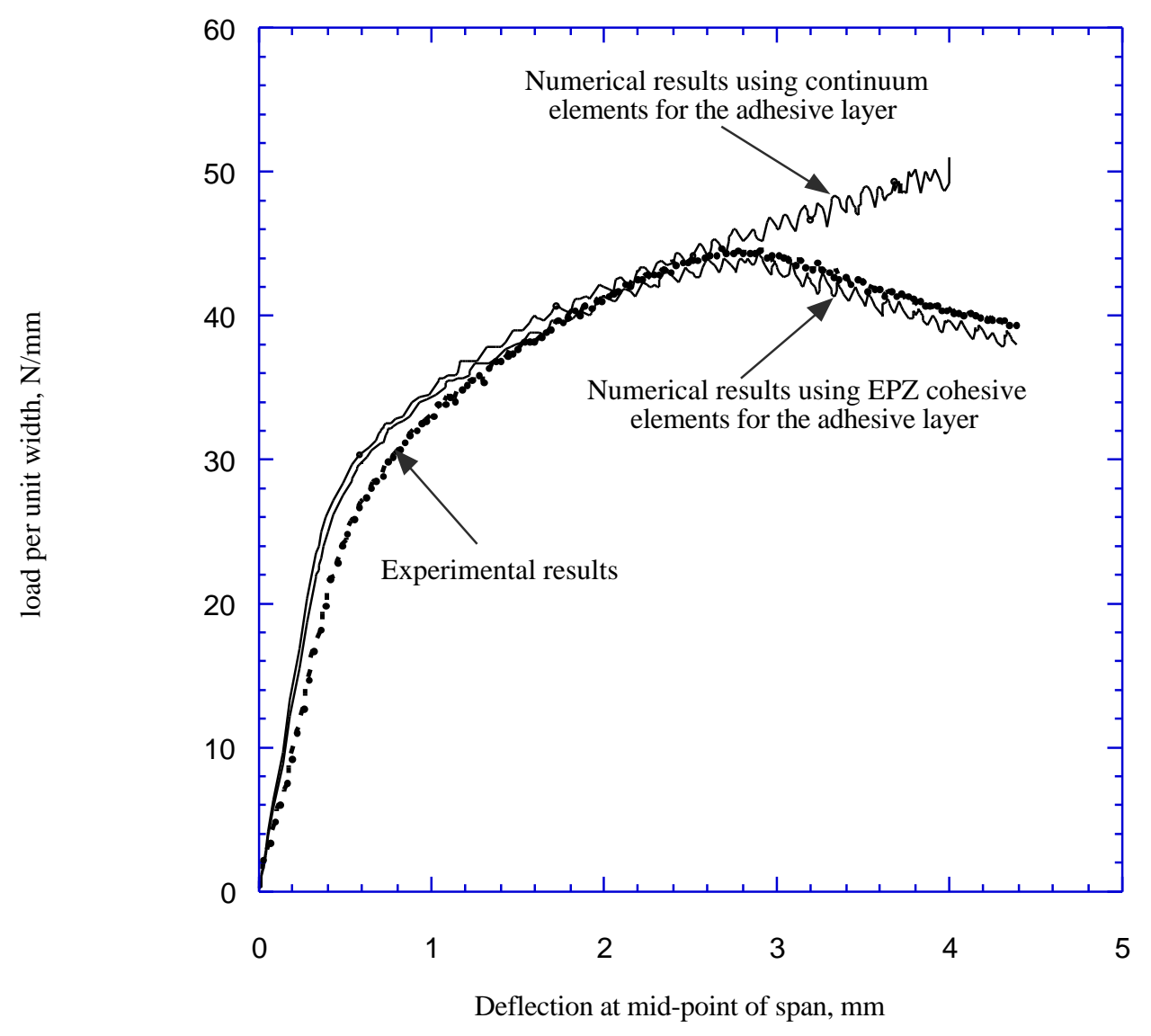

Fig. 6 


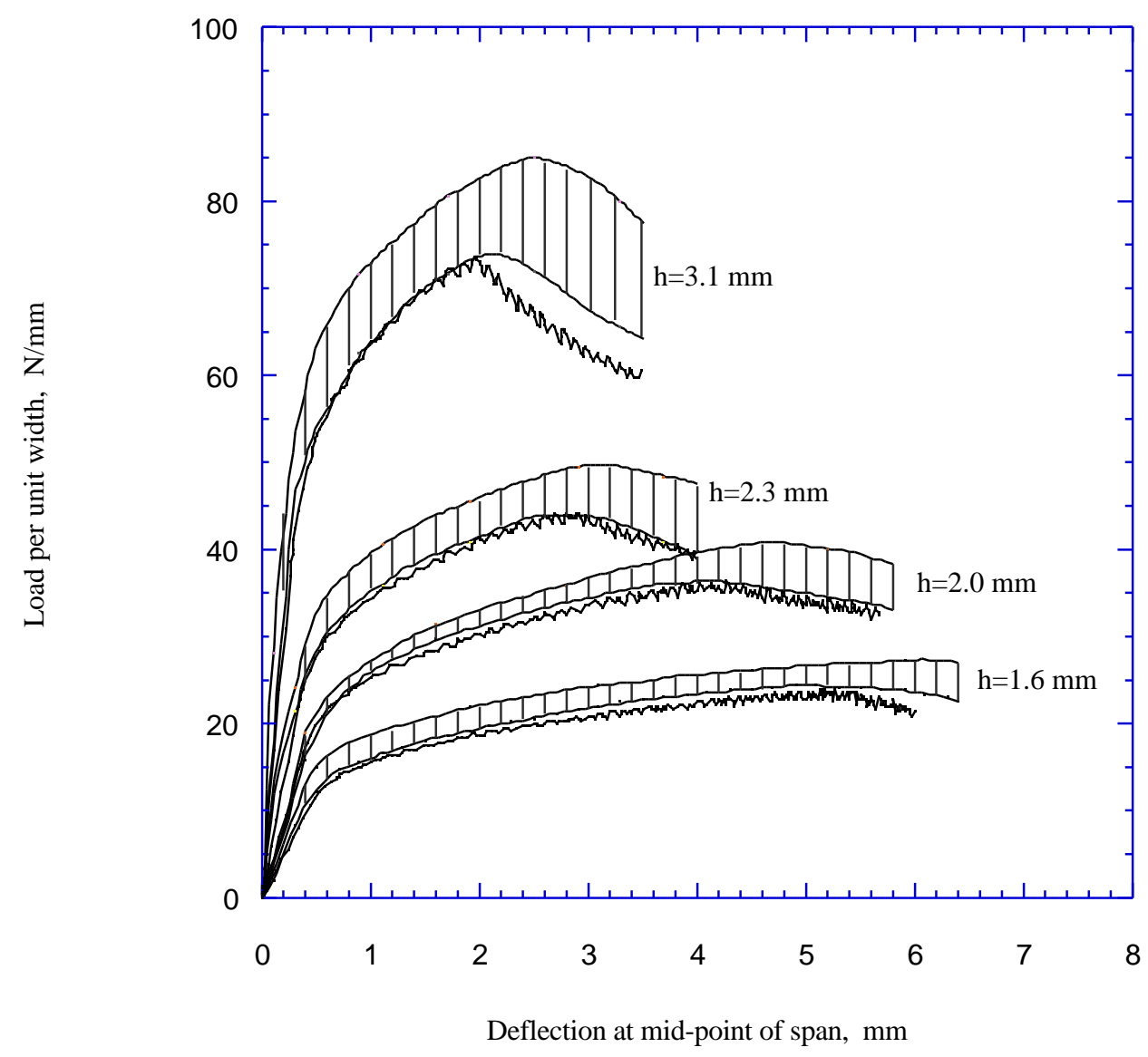

Fig. 7 


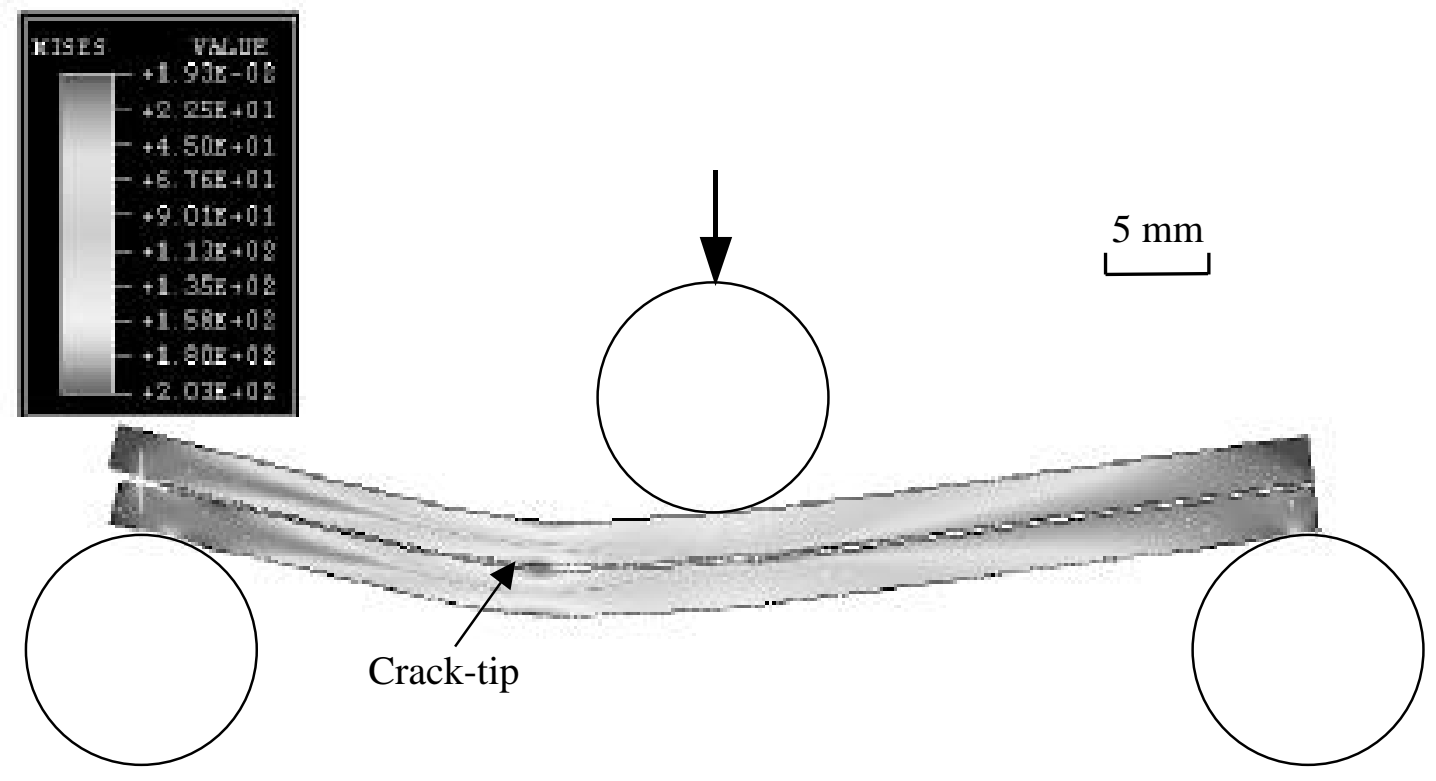

Fig. 8(a) 


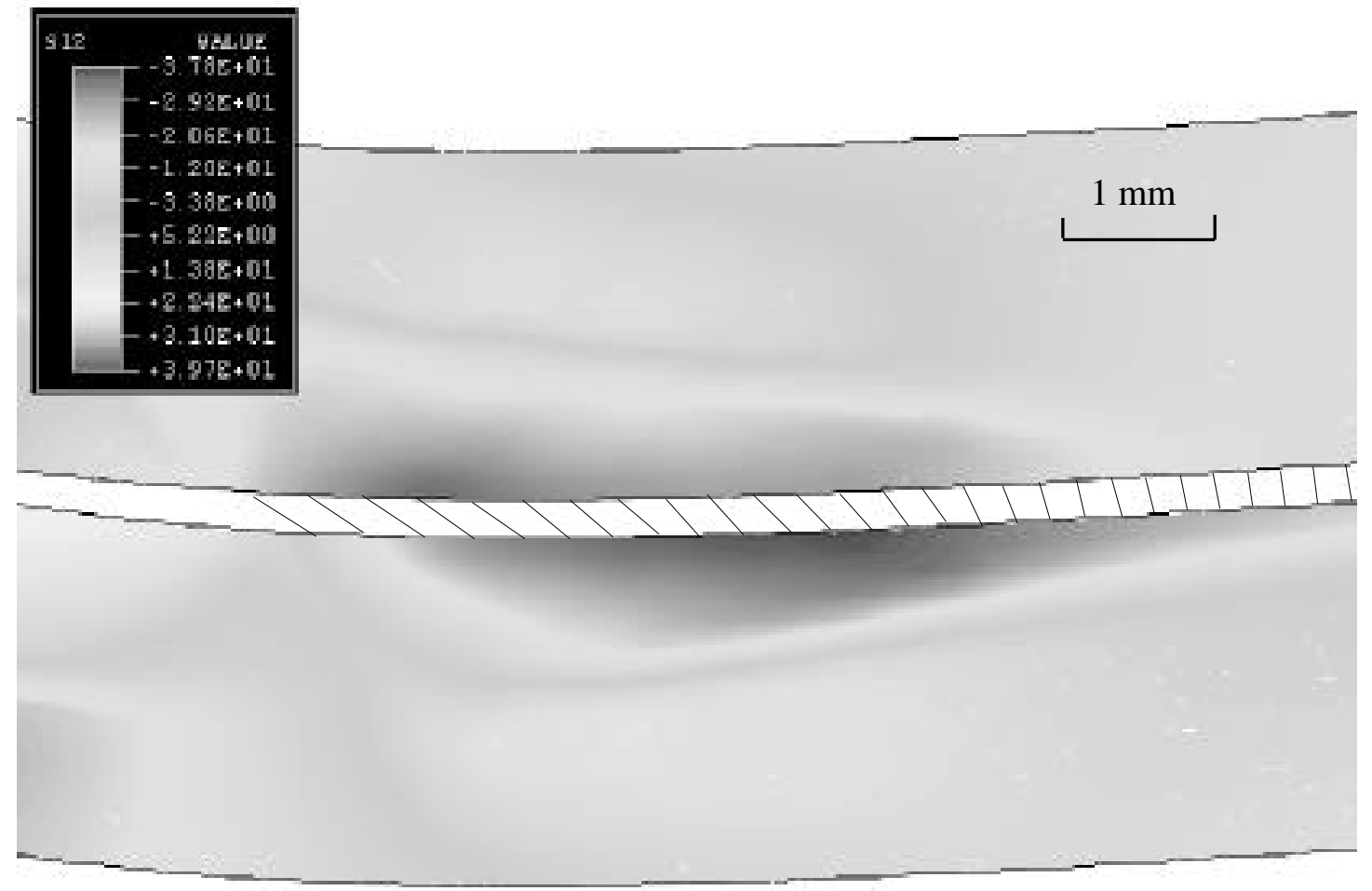

Fig. 8(b) 


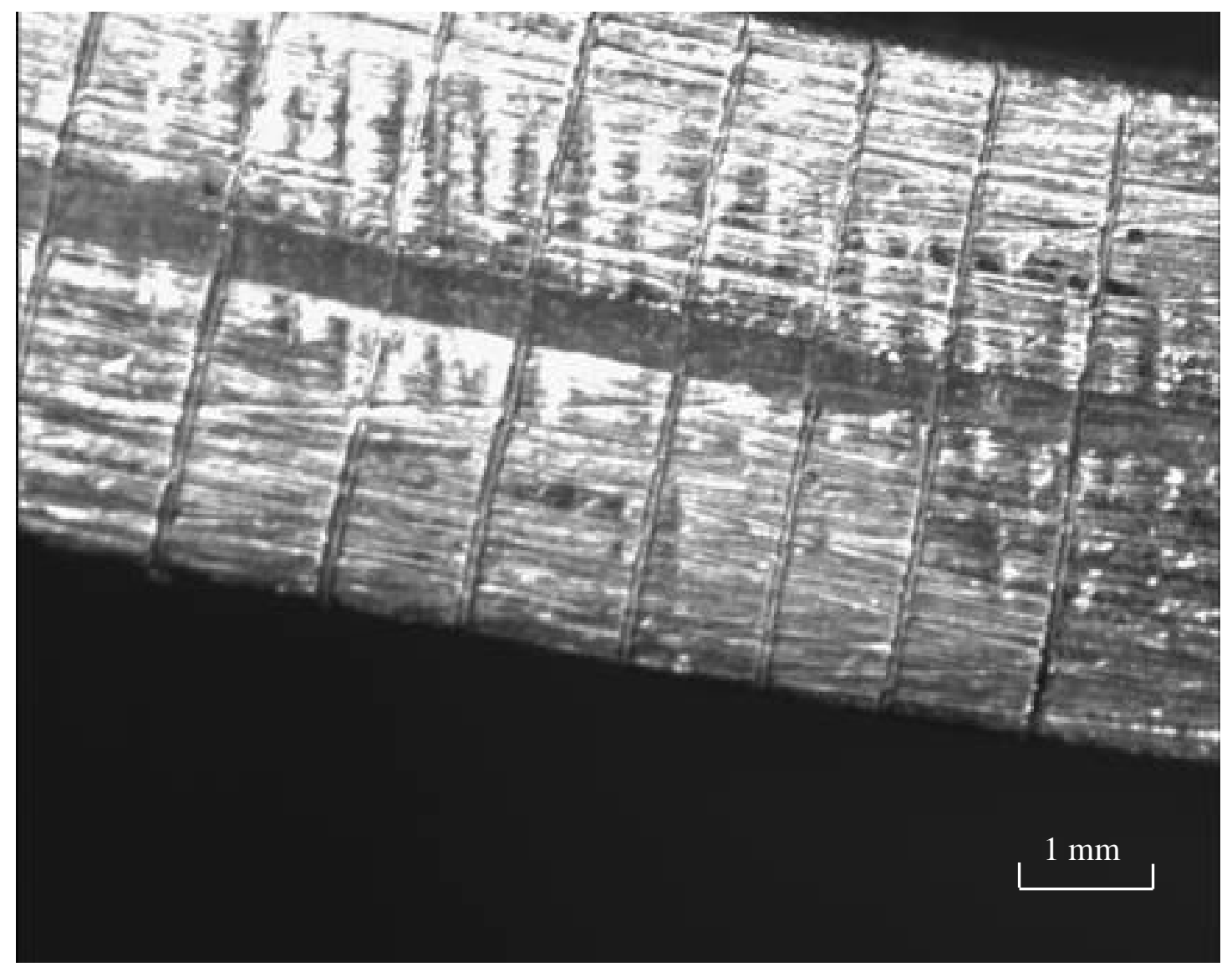

Fig. A1 


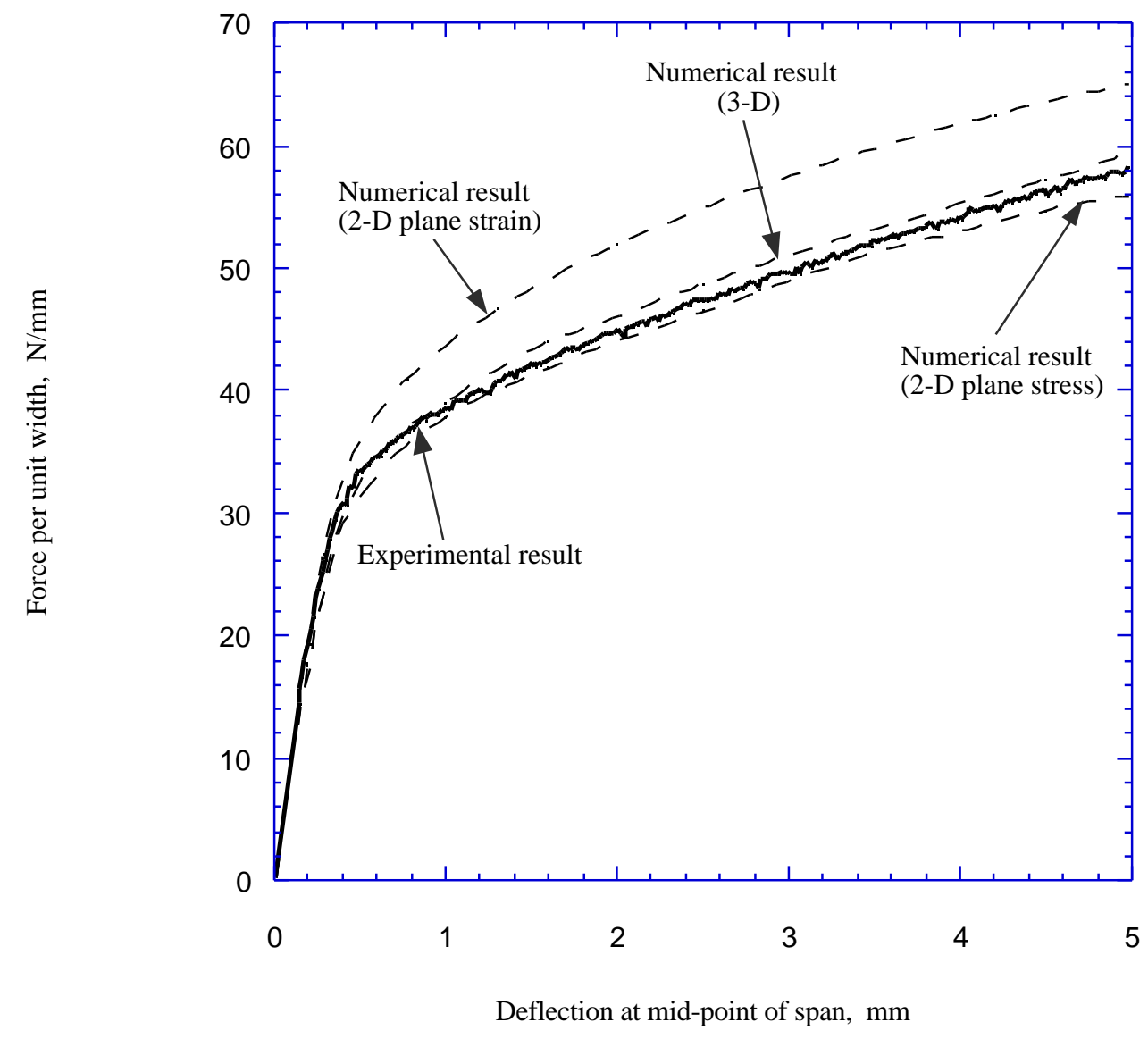

Fig. A2 